\title{
Laboratory Astrophysics Data Needed for James Webb Space Telescope Science Investigations
}

\author{
George Sonneborn \\ NASA Goddard Space Flight Center, Code 665, Greenbelt, MD 20771, USA \\ email: george.sonneborn@nasa.gov
}

\begin{abstract}
The James Webb Space Telescope (JWST) is an infrared-optimized astrophysics observatory to be launched in 2018. JWST is designed to find and study the first galaxies that formed in the early universe, to peer through dusty clouds to see star and planet formation at high spatial resolution, to obtain spectra for characterizing exoplanet atmospheres, and of rocky and icy bodies throughout the Solar System. Laboratory Astrophysics needs are related to molecular/solid data for exoplanet atmospheres and rocky/icy surfaces in the Solar System.
\end{abstract}

Keywords. Molecular data, Stars: exoplanets, Kuiper Belt, Asteroids

\section{JWST Lab Astro Needs}

JWST is a large aperture $(6.5 \mathrm{~m})$, cryogenic, infrared-optimized space observatory under construction by NASA, ESA, and CSA for launch in Oct. 2018. The JWST observatory will be placed in an Earth-Sun L2 orbit by an Ariane 5 launch vehicle provided by ESA. The observatory is designed for a 5-year prime science mission, with propellant for 10 years of science operations. The first call for proposals for JWST observations will be released in Fall 2017. Construction of the JWST observatory is nearing completion. Cryogenic testing of the full optical system is underway. The JWST instrument modes and expected sensitivity are available at http://stsci.edu/jwst/. The main laboratory astrophysics needs are in the areas of exoplanet atmospheres and icy/rocky surfaces of bodies in our Solar System (asteroids, Kuiper Belt Objects, Main Belt Comets, etc.).

Exoplanets are already known to exist with a much wider range of atmospheric conditions (temperature, pressure, stellar illumination, abundances, etc) than exist in the Solar System. JWST will observe the best exoplanets from the 1000s that have been and will be discovered. Understanding will be only as good as the atmospheric models and atomic and molecular data. Likely constituents $\left(\mathrm{CH}_{4}, \mathrm{CO}_{2}, \mathrm{CO}, \mathrm{H}_{2} \mathrm{O}, \mathrm{NH}_{3}, \mathrm{O}_{3}\right)$ are subjected to a wide range of atmospheric conditions (temperature, pressure), illumination, and photo-chemical processes. Trace species may provide clues to important processes. The high temperature and low surface gravity in hot Jupiters means their atmospheres have large scale heights. Cloud condensates are liquid or solid particles suspended in the planet's atmosphere. Optical properties (transmission, scattering, etc) of condensate materials are generally unknown for the high T,P regime in hot Jupiter atmospheres.

The $3 \mu \mathrm{m}$ region is critical for a wide range of Solar System science, primarily because key transitions of $\mathrm{OH}, \mathrm{H}_{2} \mathrm{O}, \mathrm{CO}, \mathrm{CO}_{2}$, carbonates, and hydrated minerals in this nearIR region are not observable from the ground. Kuiper Belt Objects are distant, cold, and primordial with volatiles likely retained on their surfaces. Molecular ices are very distinguishable at 2.5-5 $\mu \mathrm{m}$. See Milam et al. (2015, special JWST issue of PASP on solar system science) for details. 